\title{
URBAN EXPANSION MONITORING USING SATELLITE IMAGES BY MEANS OF DECISION LEVEL FUSION OF FUZZY CHANGE DETECTORS
}

\author{
S. Mahmoudi ${ }^{\text {a }}{ }^{*}$, M.R. Saradjian ${ }^{\text {b }}$, A. Esmaeily ${ }^{\text {a }}$, S. Vajedian ${ }^{\text {b }}$ \\ ${ }^{a}$ Dept. of Remote Sensing Engineering, Graduate University of Advanced Technology, Kerman, Iran \\ somayemahmoudi@yahoo.com, aliesmaeily@kgut.ac.ir \\ ${ }^{\mathrm{b}}$ Dept. of Surveying and Geomatics engineering, Faculty of Engineering, University of Tehran, Iran \\ sarajian@ut.ac.ir, s_vajedian@yahoo.com
}

KEY WORDS: Change Detection, Urban Expansion, Fuzzy, Decision Fusion, ASTER Image

\begin{abstract}
:
This study investigates urban expansion using satellite images by means of decision level fusion of two different fuzzy change detectors. The aim of this study is taking the advantage of effects of various change detection techniques. These techniques are differentiated by the way they use the dataset which their fusion could lead us to gain complementary outputs and ultimately achieve higher accuracy in change detection. This study focuses on the use of this framework specifically for urban region expansion monitoring. Here outputs of two fuzzy change detectors based respectively on post classification comparison and spectral-temporal combined analysis methods of multi-temporal ASTER images are fused by using Sugeno fuzzy integral operator. Accuracy assessment that has been done by available land cover maps for fuzzy post classification comparison combined fuzzy spectraltemporal analysis and their combination technique has shown improvement of change detection accuracy over each single fuzzy change detector in urban expansion application.
\end{abstract}

\section{INTRODUCTION}

Change detection is a major challenge in many applications such as: natural disaster, land use / land cover changes, changes in rivers and lakes, shoreline changes and changes in urban areas. Urban region expansion monitoring is one of the important applications of change detection. Remote sensing is a very powerful tool for land use/land cover change monitoring with appropriate temporal resolution and low cost. A large number of various techniques have already been developed for change detection in remotely sensed imagery. Methods that are based on classification are important since they make an optimal use of all spectral bands, minimize impacts of atmospheric, sensor and environmental differences between multi-temporal images and provide a complete matrix of change information ( $\mathrm{Lu}$ et al., 2004). The features of the urban area have high variability and each pixel can contain many land cover types, so appropriation of each pixel to only one class is not logical. In such cases, employment of fuzzy classification algorithms are useful. In fuzzy change detection, several studies have been conducted. Deer, proposed a fuzzy approach to post classification change detection technique in remotely sensed imagery, that focuses on its use for environmental monitoring (Deer, 1999). Hedge, explores the use of fuzzy operators in identifying and quantifying changes, nature and direction of changes occurring in land cover (Hedge, 2003). Nemmour and Chibani, proposed a fuzzy fusion framework to improve change detection in remotely sensed data (Nemmour and Chibani, 2004). Colditz et al. presented bi-annual change detection technique builds directly on the fuzzy classification result and presented the change in land cover composition for each class or each pixel (Colditz et al., 2008). Lee has utilized a spatial region growing segmentation and a classification using fuzzy membership vectors to detect the changes in the images observed at different dates (Lee, 2010). Ghosh et al. proposed a context-sensitive technique for unsupervised change detection in multi-temporal remote sensing images. Their technique is based on fuzzy clustering approach and takes care of spatial correlation between neighboring pixels of the difference image produced by comparing two images acquired on the same geographical area at different times (Ghosh et al., 2011). Mishra et al. used two fuzzy clustering algorithms, namely fuzzy cmeans (FCM) and Gustafson-Kessel clustering (GKC) along with local information for unsupervised change detection in multi-temporal remote sensing images. In conventional FCM and GKC no spatio-contextual information is taken into account and thus the result is not so much robust to small changes. Since the pixels are highly correlated with their neighbors in image space (spatial domain), incorporation of local information enhances the performance of the algorithms. So they introduced a new technique for incorporation of local information (Mishra et al., 2012). Different change detectors produce different results since they handle the data differently. In return, they offer complementary information because the sets of patterns misclassified do not necessarily overlap (Kittler et al., 1998). Generally, change map from multi-temporal remote sensing images using any single method or single kind of data source may contain a number of omission/commission errors, degrading the detection accuracy to a great extent. There are some investigations deployed to get interesting and valuable findings about fusion approaches incorporated into change detection process (Du et al., 2013). Le Hegarat-Mascle et al. applied decision level fusion based on Dempster-Shafer (D-S) evidence theory to change detection from multispectral imagery and obtained the fusion of multi-index change detection results (Le Hegarat-Mascle et al., 2006). Nemmour and Chibani used two combination rules, namely, Fuzzy Integral and Attractor Dynamics to combine different SVM-based change detection techniques for mapping urban growth in the Algerian capital (Nemmour and Chibani, 2006). Bovolo et al. analyzed the impacts of different pan-sharpening (PS) techniques on change detection, and ranked these PS approaches according to their impacts on change detection performance by using a novel unsupervised similarity measure (Bovolo et al., 2010). Although change maps obtained from pan-sharped images spatially enhanced but affected by errors that arise from fusion process. 
So in order to investigate the impacts of different information fusion techniques on change detection, $\mathrm{Du}$ et al. proposed a sequential fusion strategy combining pan-sharpening with decision level fusion to change detection from multi-temporal remotely sensed images (Du et al., 2013). This study use a fusion framework that there in outputs of two fuzzy change detectors based respectively on post classification comparison and spectral-temporal combined analysis methods of multitemporal data are fused by using Sugeno fuzzy integral operator. Nemmour and Chibani used this framework for change detection in a coastal region by SPOT images. This study focuses on use of this framework for change detection in urban region by ASTER images.

\section{STUDY AREA}

The study area is located in the northern part of the city of Karaj in Iran.

\section{DATA}

In order to carry out the experimental analysis aimed to assess the effectiveness of the selected approach, we considered two multi-temporal remote sensing data sets with the same spectral, radiometric and spatial resolution corresponding to geographical areas of Karaj, Iran. The spatial resolution of the sensor (ASTER) is 15 meters. Each pixel thus represents an area of $15 \mathrm{~m}-15 \mathrm{~m}$. In order to reduce the effects of sun angle, seasonal and phonological differences, they've been acquired nearly in the same date but with 11 years time interval. Figs. 1(a) and (b) show false color composite of the 2001 and 2012 images, respectively.
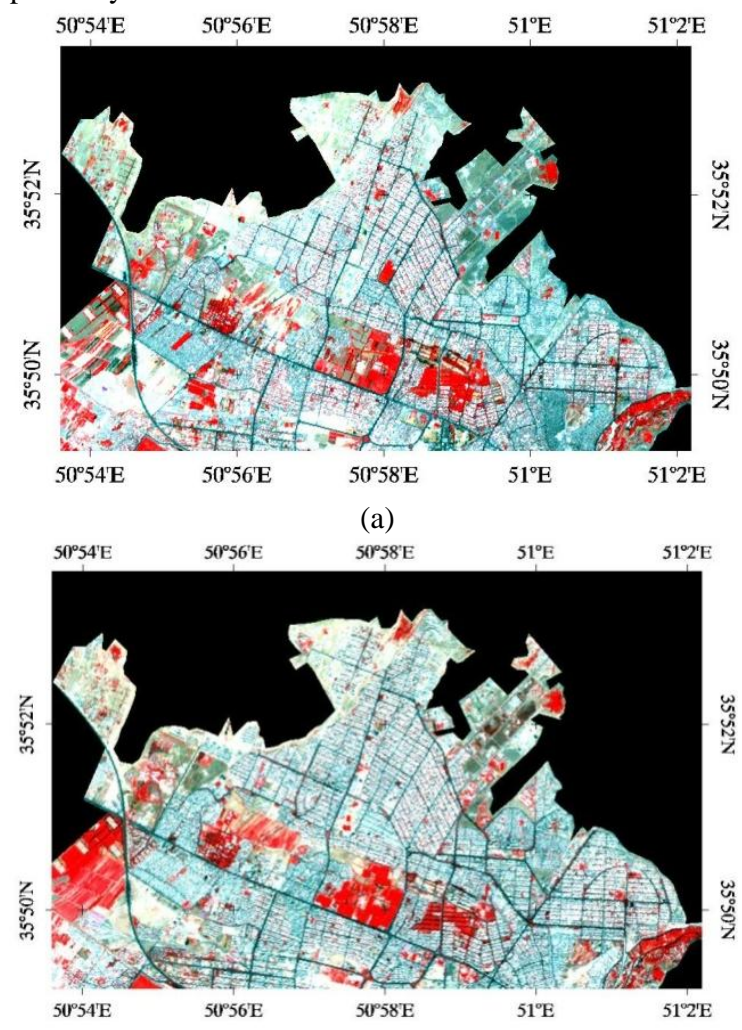

(b)

Figure 1. Image of study area. (a) false color composite of the ASTER image acquired in July 2001, (b) false color composite of the ASTER image acquired in September 2012

\section{METHODOLOGY}

\subsection{Pre-processing}

Geometric correction to correct the geometric distortions including internal and external distortions and radiometric correction to reconstruct physically calibrated values by correcting the spectral distortions caused by sensors, sun angle, topography and the atmosphere are two essential pre-processing for change detection process. Obviously in pixel based techniques, the geometric registration should be performed accurately; otherwise, the change detection could be erroneous. Therefore, here, in pre-processing step, precise geometric correction and radiometric calibration have been performed.

\subsection{Change detection}

Due to the importance of nature of changes in urban expansion application, the change detection techniques which are based on classification approaches are used in this study. Because of the high variability in the features of the urban area, each pixel can contain many land cover types, so appropriation of each pixel to only one class is not logical. For this reason, fuzzy classification based techniques are used in this study. For implementation of fuzzy post classification comparison technique, each image has been individually classified. Classification performed by using Mahalonobis distance fuzzy classifier and the degree of membership of each pixel in each of the classes of interest was calculated according to the following equation:

$$
\mu_{(k, 1) i}=\frac{\left(\frac{1}{d_{(k, l) i}^{2}}\right)^{r}}{\sum_{j, p=1}^{c}\left(\frac{1}{d_{(j, p) i}^{2}}\right)^{r}}
$$

Where $\mu_{\mathrm{ik}}$ is the membership degree of pixel $\mathrm{x}_{\mathrm{i}}$ in class $\mathrm{k}, \mathrm{c}$ is number of classes, $r$ is fuzzification parameter. $d_{i k}$ is Mahalonobis distance between pixel $\mathrm{x}_{\mathrm{i}}$ and class $\mathrm{k}$.

Then, with fuzzy aggregation Min operator, the fuzzy membership of each pixel in each change class has been calculated as follows:

$$
\mu_{(\mathrm{k}, 1) \mathrm{i}}=\min \left(\mu_{\mathrm{ki}}\left(\mathrm{t}_{1}\right), \mu_{\mathrm{li}}\left(\mathrm{t}_{2}\right)\right)
$$

Where $\mu_{(k, l) i}$ is fuzzy membership degree of pixel $x_{i}$ in change class $(k, l), \mu_{\mathrm{ki}}(\mathrm{t} 1)$ is fuzzy membership degree of pixel $\mathrm{x}_{\mathrm{i}}$ in class $\mathrm{k}$ at the date1, $\mu_{\mathrm{li}}(\mathrm{t} 2)$ is fuzzy membership degree of pixel $\mathrm{x}_{\mathrm{i}}$ in class 1 at the date2.

In order to implement combined fuzzy spectral-temporal analysis, fuzzy classifier has been used to classify the spectral bands of two images simultaneously. In this way, the fuzzy membership of each pixel in each change class has been calculated according to the following equation: 


$$
\mu_{(k, 1) i}=\frac{\left(\frac{1}{d_{(k, 1) i}^{2}}\right)^{r}}{\sum_{j, p=1}^{c}\left(\frac{1}{d_{(j, p) i}^{2}}\right)^{r}}
$$

\subsection{Decision level fusion}

Within aggregation operators, fuzzy integrals are known to be one of the most powerful and flexible functions as they permit the aggregation of information under different assumptions on the independence of the information sources. Fuzzy integrals combine the data supplied by several information sources according to a fuzzy measure. This fuzzy measure, that (using Artificial Intelligence terminology) represents the background knowledge on the information sources, is a set function from the set of information sources into an appropriate domain (e.g. the $[0,1]$ interval).Typically, this fuzzy measure represents the importance or relevance of the sources when computing the aggregation (Vicenc Torra, 2006).

If $Y=\left\{y_{1}, y_{2}, \ldots, y_{n}\right\}$ is a finite set, assume $h: Y \rightarrow[0,1]$ is a function as $h\left(y_{1}\right) \geq h\left(y_{2}\right) \geq \ldots \geq h\left(y_{n}\right)$, in this case, Sugeno fuzzy integral of a function $\mathrm{h}$ with respect to fuzzy measure $\mathrm{g}$ is defined as:

$$
I_{S}=\int \operatorname{hog}=\operatorname{Max}_{i=1}^{n}\left[\operatorname{Min}\left(h\left(y_{i}\right), g\left[A_{i}\right]\right)\right]
$$

Where $A_{i}=\left\{y_{1}, y_{2}, \ldots, y_{n}\right\}$ and $g\left(A_{i}\right)$ computed as follows:

$$
\begin{aligned}
& g\left(A_{1}\right)=g\left(\left\{y_{1}\right\}\right)=g^{1} \\
& g\left(A_{i}\right)=g{ }^{i}+g\left(A_{i-1}\right)+\lambda g^{i} g\left(A_{i-1}\right) \quad 1<\mathrm{i}<\mathrm{n}
\end{aligned}
$$

$\lambda$ can be computed by solving $\mathrm{g}(\mathrm{Y})=1$ according to the following equation:

$$
\lambda+1=\underset{\mathrm{i}=1}{\amalg}\left(1+\lambda \mathrm{g}^{\mathrm{i}}\right)
$$

Where $\lambda \in(-1,+\infty)$ and $\lambda \neq 0$

Then, in the last step of mentioned framework, results that achieved from post classification comparison and spectraltemporal analysis techniques have been fused by means of Sugeno fuzzy integral operator with respect to fuzzy measures that represent the difference of operation between them.

\section{IMPLEMENTATION}

First, in pre-processing step, precise geometric correction with accuracy of sub-pixel level by combined method has been done. Also in order to do radiometric calibration, normalization between two images has been performed. After precise geometric correction and radiometric correction of each date image, for implementation of fuzzy post classification comparison technique, each image has been individually classified to four classes including urban, soil, orchard and cultivation. According to four primary mentioned classes, eight change and no-changed classes was considered. Then, the fuzzy membership of each pixel in each change and no-changed class has been calculated. Output of defuzzification step that performed by Max operator, shown in Fig. 2.

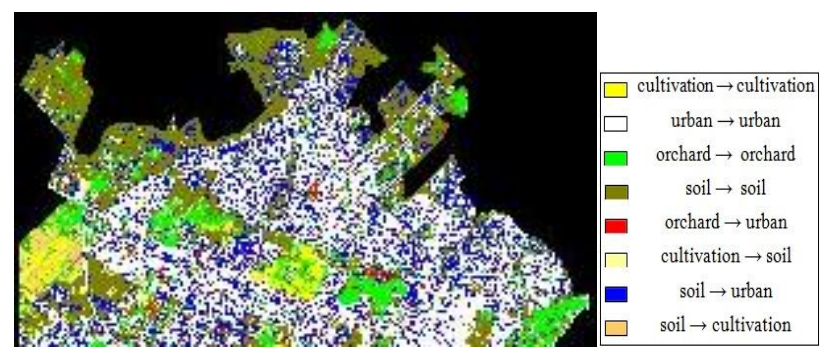

Figure 2. Output of defuzzification step of post classification comparison technique

Then, change detection performed by spectral-temporal combined analysis. Output of defuzzification step shown in Fig. 3.

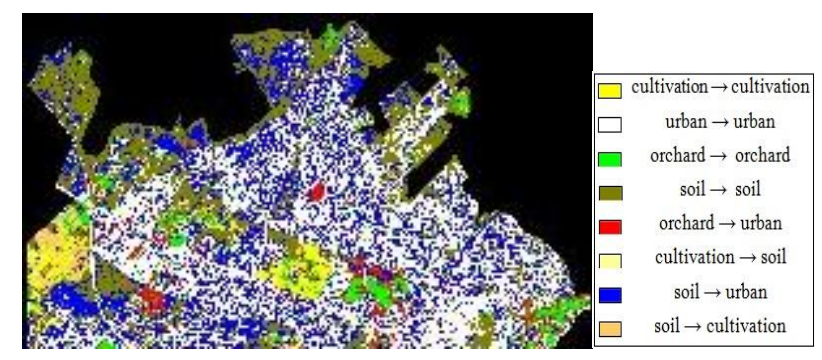

Figure 3. Output of defuzzification step of spectral-temporal combined analysis

Accuracy assessment has been done by available land cover maps for fuzzy post classification comparison and combined fuzzy spectral-temporal analysis. Calculated kappa coefficients for these methods are 0.91 and 0.90 respectively.

Then, in the last step, results that achieved from post classification comparison and spectral-temporal analysis techniques have been fused by means of Sugeno fuzzy integral operator with respect to fuzzy measures that construct based on user accuracy of each of mentioned methods in each class. Output of defuzzification step shown in Fig. 4.

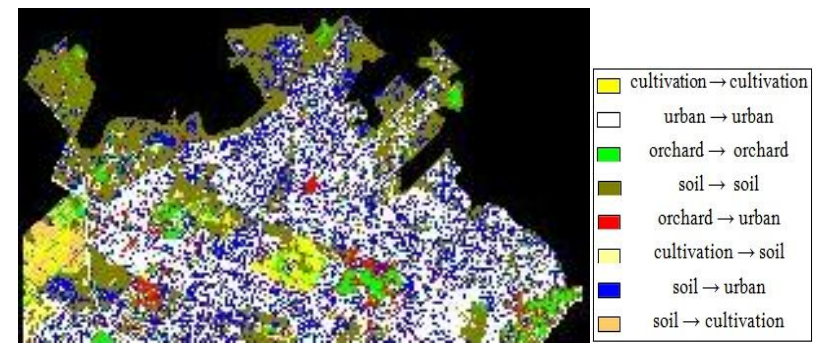

Figure 4. Output of defuzzification step of spectral-temporal combined analysis of Sugeno fuzzy integral operator

Accuracy assessment has been done by available land cover maps for outputs of this combination method. Calculated kappa coefficient is 0.94 . 


\section{DISCUSSION AND CONCLUSION}

The accuracy assessment was derived based on the available land cover maps for fuzzy post classification comparison, combined fuzzy spectral-temporal analysis. Their combination techniques has shown improvement of change detection accuracy over each single fuzzy change detector in urban expansion application. This is because: 1) these techniques are different in the way they use the dataset, so their outputs are complementary, 2) on the other, in spectral-temporal combined analysis as a single classification is performed in one step, it avoids combining respective inaccuracies that are common with post classification comparison procedures (Desclee et al., 2006). Therefore their fusion leaded us to achieve higher accuracy in change detection. In the study area two classes including soil $\rightarrow$ urban and orchard $\rightarrow$ urban are classes that show the urban region expansion. Outputs analysis showed that urban region growing in study area first is related to class soil $\rightarrow$ urban that is due to settlement in borders of city and construction growth inside the city. Second related to class orchard $\rightarrow$ urban that is due to parks and building construction instead orchards. So this study proved efficiency of mentioned framework for change detection in urban region by ASTER images.

\section{ACKNOWLEDGMENT}

The authors would like to thank Dr. Barat Mojaradi for the contribution given in the data preparation of this work.

\section{REFERENCES}

Bovolo, F., Bruzzone, L., Capobianco, L., Garzelli, A., Marchesi, S., Nencini, F., 2010. Analysis of the Effects of Pansharpening in Change Detection on VHR Images, IEEE Geosci. Remote Sens. Lett., vol. 7, no. 1, pp. 53-57.

Colditz, R. R., Schmidt, M., Dech, S., 2008. A methodology for advanced change detection with fuzzy image classification, in 5th International Conference on Electrical Engineering, Computing Science and Automatic Control, 2008. CCE 2008, 2008, pp. 520-524.

Deer, P., 1999. Digital Change Detection in Remotely Sensed Imagery Using Fuzzy Set Theory, PHD thesis, Department of Geography and Department of Computer Science, University of Adelaide, Australia.

Desclée, B., Bogaert, P., Defourny, P., 2006. Forest change detection by statistical object-based method," Remote Sens. Environ., vol. 102, no. 1-2, pp. 1-11.

Du, P., Liu, S., Xia, J., Zhao, Y., 2013. Information fusion techniques for change detection from multi-temporal remote sensing images, Inf. Fusion, vol. 14, no. 1, pp. 19-27.
Ghosh, A., Mishra, N. S., Ghosh, S., 2011. Fuzzy clustering algorithms for unsupervised change detection in remote sensing images, Inf. Sci., vol. 181, no. 4, pp. 699-715.

Hegde, S.,2003. Modelling Land Cover Change: A Fuzzy Approach, Master of Science thesis, International Institute for Geo-information Science and Earth Observation, Netherlands.

Kittler, J., Hatef, M., Duin, R. P. W., Matas, J., 1998. On combining classifiers, IEEE Trans. Pattern Anal. Mach. Intell., vol. 20, no. 3, pp. 226-239.

Lee, Sang-Hoon., 2010. Automated change detection in land cover pattern using region growing segmentation and fuzzy vector, presented at the A special joint symposium of ISPRS Technical Commission IV \& AutoCarto in conjunction with ASPRS/CaGIS 2010 Fall Specialty Conference, Orlando, Florida.

Le Hegarat-Mascle, S., Seltz, R., Hubert-Moy, L., Corgne, S., Stach, N., 2006. Performance of change detection using remotely sensed data and evidential fusion: comparison of three cases of application," Int J Remote Sens, vol. 27, pp. 35153532 .

Lu, D., Mausel, P., Brondízio, E., and Moran, E., 2004. Change detection techniques. Int. J. Remote Sens., vol. 25, no. 12, pp. 2365-2401, 2004.

Mishra, N. S., Ghosh, S., Ghosh, A., 2012. Fuzzy clustering algorithms incorporating local information for change detection in remotely sensed images, Appl. Soft Comput., vol. 12, no. 8, pp. 2683-2692.

Nemmour, H., Chibani Y., 2004. Change detector combination in remotely sensed images using fuzzy integral, Int. Jour-Nal Signal Process., vol. 1, no. 4, pp. 175-181.

Nemmour, H., Chibani, Y., 2006. Multiple support vector machines for land cover change detection: An application for mapping urban extensions," ISPRS J. Photogramm. Remote Sens., vol. 61 , no. 2 , pp. 125-133.

Vicenç Torra, Y. N., 2006. The interpretation of fuzzy integrals and their application to fuzzy systems.," Int J Approx Reason., vol. 41, pp. 43-58. 